\title{
Bei akutem Herzinfarkt: nur den Verschluss oder alle Stenosen dilatieren?
}

\section{Soll man nur das für den akuten Infarkt verantwortliche Gefäß dila- tieren oder gleich alle erreichbaren Koronarstenosen? Zwei Studien belegen einmal mehr die These: weniger aggressiv ist besser.}

- In der Studie von Karnowski et al. wurde bei Patienten mit akutem Herzinfarkt und multiplen Koronarstenosen entweder zunächst nur das für den Infarkt verantwortliche Gefäß dilatiert, andere Stenosen dann 6 bis 51 (im Mittel 30) Tage später $(n=393)$ oder aber gleichzeitig bei der ersten Sitzung dilatiert $(n=275)$. Bei einzeitigem Vorgehen, d.h. sofortiger Dilatation aller relevanten Stenosen waren Gesamtmortalität (9,2 vs. $2,3 \%)$ und kardiale Mortalität (6,2 vs. $2,0 \%)$ höher, Stentthrombosen (5,7 vs. $2,3 \%$ ) und kardiale Komplikationen 18,1 vs. $13,4 \%$ ) häufiger. Die Beschränkung auf das Infarktgefäß bei der ersten Sitzung und elektive Versorgung der anderen Koronargefäße zu einem späteren Zeitpunkt war ebenfalls günstiger als die sofortige Dilatation aller Stenosen.

In einer Metaanalyse haben Vlaar und Mitarb. vier prospektive und 16 retrospektive Studien mit 40280 Patienten ausgewertet und kommen zu dem gleichen Schluss: Bei zweizeitiger Dilatation sind Kurz- und Langzeitmortalität niedriger als bei PTCA mehrerer Koronarien. Die sofortige Mehrgefäßdilatation hat kurz- und langfristig die höchste Mortalität.

\section{Kommentar}

Patienten mit akutem Myokardinfarkt haben häufig Stenosen auch an anderen Koronararterien. Allerdings kann der Kardiologe beim Herzkatheter in der Regel zuverlässig das für die Ischämie verantwortliche Gefäß identifizieren. Da bieten sich naturgemäß zwei Strategien an: im akuten Stadium nur das verantwortliche Gefäß aufdehnen und den Eingriff an den anderen Gefäßen zurückstellen und vom weiteren Verlauf abhängig machen oder aber - da der Katheter nun schon einmal liegt - auch die anderen Stenosen in der gleichen Sitzung aufzuweiten. Die Ergebnisse stützen die Empfehlungen der Fachgesellschaften, die die Mehrgefäßintervention nicht als Standard, sondern nur für Ausnahmen, z. B. für Patienten im Schock, vorsehen.

In den letzten Jahrzehnten tendierte die Medizin zu möglichst intensiven und aggressiven Maßnahmen und ehrgeizigen Behandlungszielen. Das Spektrum reichte

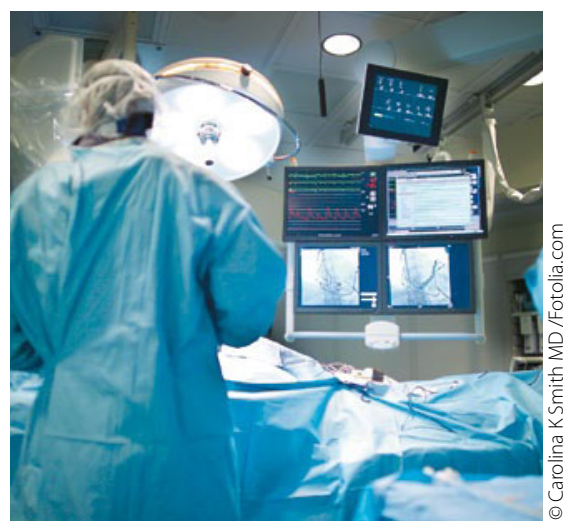

Eine Koronarstenose kommt selten allein.

von der strengen Blutzuckereinstellung bei Diabetikern bis zur Radikalbehandlung des Mammakarzinoms. In vielen Bereichen erfolgte eine Kehrtwende: Aus dem , je mehr und je intensiver, umso besser" wird immer häufiger ein "weniger ist mehr".

H. HolzGREVE =

\footnotetext{
- R. Karnowski et al

Prognostic impact of staged versus "one-time" multivessel percutaneous intervention in acute myocardial infarction.

P. J. Vlaar et al.

Culprit vessel only versus multivessel and staged percutaneous coronary intervention for multivessel disease in patients presenting with ST-segment elevation myocardial infarction. J. Am. Coll. Cardiol. 58 (2011) 692-711
} 